\title{
Leitfähigkeit von Hexan bei Einwirkung von $\gamma$-Strahlen und ihre Bedeutung für die Dosimetrie *
}

\author{
A. JAHNS * und W. JACOBI \\ Hahn-Meitner-Institut für Kernforschung, Berlin, Abteilung Strahlenphysik \\ (Z. Naturforschg. 21 a, 1400-1410 [1966] ; eingegangen am 2. April 1966)
}

\begin{abstract}
The influence of primary and secondary recombination on the $\gamma$-ray-induced conductivity of liquid n-hexane is studied experimentally and theoretically. Conductivity measurements were made with a parallel-plate ionization chamber, varying the electrical field, the electrode distance and the dose rate. The primary recombination can be explained with a theory developed by ONSAGER for the recombination of single ion pairs in dense gases, whereas JAFFE's theory of column ionization is not applicable. Independent of the ion mobilities, for hexane in absence of an electric field a $G$-value of $0,13 \pm 0,01$ for the ion pairs escaping primary recombination is obtained; this corresponds to a mean escape probability of $3 \%$ and a mean effective initial distance of the ions in an ion pair of $80 \mathrm{~m} \mu$. Finally, the applicability of liquid-filled ionization chambers for dosimetry is discussed.
\end{abstract}

Die durch ionisierende Strahlen hervorgerufene Leitfähigkeit von Flüssigkeiten und Gasen wird durch die Rekombination der strahleninduzierten Ionen begrenzt. Dabei sind zwei Rekombinationsphasen zu unterscheiden: die Primärrekombination - in der Literatur häufig auch Anfangs- oder bevorzugte Rekombination genannt - und die zeitlich sich daran anschließende Sekundär- oder Volumenrekombination. Die Primärrekombination ist durch den Entstehungsort der Ionen bedingt und spielt sich im Bereich der Bahn des ionisierenden Teilchens ab. Bei der Sekundärrekombination handelt es sich um die Rekombination von Ionen, die der Primärrekombination entgangen und ins Volumen hinausdiffundiert sind.

Hinsichtlich der Wirksamkeit der Primärrekombination besteht ein erheblicher Unterschied zwischen Flüssigkeiten und Gasen. Die räumliche Verteilung der Ionen längs der Bahn eines ionisierenden Teilchens ist zwar in beiden Zuständen geometrisch ähnlich, jedoch ist sie in Flüssigkeiten infolge der höheren Dichte entsprechend komprimiert. So liegt z. B. der mittlere Anfangsabstand der Ionen eines Ionenpaares in Gasen von Atmosphärendruck bei etwa $5 \mu$, was einer Соицомв-Bindungsenergie von

* Ein Teil dieser Untersuchung war Gegenstand ener Diplomarbeit von A. JAHNS an der Freien Universität Berlin.

* Jetzt: Institut für Reaktorsicherheit, Köln; z. Zt. NRTS Idaho Falls, USA.

1 G. JAFFe, Ann. Phys. 42, 303 [1913].

2 I. Adamczewsin, Selected Topics in Radiation Dosimetry, IAEA, Wien 1961, 191-201.

3 A. O. Allen u. A. Hummel, Discussions Faraday Soc. 36, 95 [1963]. etwa $5 \cdot 10^{-16} \mathrm{erg}$ entspricht. Letztere ist somit klein gegenüber der thermischen Energie $k T$ der Ionen von etwa $5 \cdot 10^{-14}$ erg bei Zimmertemperatur. Infolgedessen spielt die Primärrekombination in Gasen praktisch keine Rolle. In Flüssigkeiten ist hingegen infolge der kleineren Ionenabstände das CouLomBPotential größer oder vergleichbar mit der thermischen Energie. Dies hat zur Folge, daß die meisten längs der Teilchenbahn gebildeten Ionenpaare innerhalb sehr kurzer Zeit nach ihrer Entstehung unter Bildung angeregter neutraler Moleküle durch Primärrekombination verlorengehen. Im Gegensatz zu Gasen wird daher in Flüssigkeiten auch bei den höchsten erreichbaren Feldstärken kein Sättigungsstrom erreicht.

Ausgehend von den grundlegenden Untersuchungen von $\mathrm{J}_{\mathrm{AFFE}}{ }^{1}$ wurden zahlreiche Messungen der durch Röntgen- und $\gamma$-Strahlen bewirkten Leitfähigkeit in dielektrischen Flüssigkeiten durchgeführt; in neuerer Zeit sind vor allem die Arbeiten von Adamczewsir ${ }^{2}$, Allen et al. ${ }^{3}$, Blanc et al. ${ }^{4,5}$, Freeman et al. ${ }^{6,7,8}$, Januszaitis ${ }^{9}$ und Ullmaier ${ }^{10}$ zu erwähnen. Dabei wurde - mit Ausnahme von FreEмan - zur Deutung der Primärrekombination stets die JAfFesche Theorie der Kolonnenionisation ${ }^{1}$ her-

${ }^{4}$ D. Blanc, J. Mathieu u. J. Boyer, Nuovo Cim. 19, 929 [1961].

j D. Blanc J. Mathieu u. P. Vermande, Dielectrics 1, 215 [1964].

6 G. R. Freeman, J. Chem. Phys. 39, 988 [1963].

7 G. R. Freeman, J. Chem. Phys. 39, 1580 [1963].

8 G. R. Freeman u. J. M. Fayadh, J. Chem. Phys. 43, 86 [1965].

9 A. Januszaitis, Acta Phys. Polon. 24, 809 [1963].

10 H. Ullmaier, Z. Phys. 178, 44 [1964]. 
angezogen, obwohl die Voraussetzungen dieser Theorie für Strahlenarten geringer LET (linear energy transfer) nicht erfüllt sind, worauf bereits JAFFE hingewiesen hat. Darauf ist auch zurückzuführen, daß sich bei Anwendung dieser Theorie viel zu hohe Werte für den mittleren Energieverlust $W$ pro gebildetem Ionenpaar in Flüssigkeiten ergeben 10, 11. Im Widerspruch zur Theorie von JAFFE ${ }^{1}$ und ihrer Modifikation durch Kramers ${ }^{12}$ steht ferner die beobachtete Abhängigkeit der Leitfähigkeit von der Quantenenergie ${ }^{9}$. Auch die Anwendung der von LeA ${ }^{13}$ auf den Fall der Clusterionisation erweiterten Form dieser Theorie stößt auf Schwierigkeiten.

In der vorliegenden Arbeit wird die Primär- und Sekundärrekombination in dielektrischen Flüssigkeiten in Anlehnung an die Ionenrekombination in Gasen diskutiert. Dabei wird zur Deutung der Primärrekombination eine von ONSAGER ${ }^{14}$ entwickelte Theorie der Rekombination einzelner Ionenpaare in komprimierten Gasen herangezogen, auf deren Anwendbarkeit auf dielektrische Flüssigkeiten bereits FreEman $^{7,8}$ hingewiesen hat. Das Ergebnis dieser theoretischen Analyse wird verglichen mit experimentellen Untersuchungen der durch $\mathrm{Co}^{60}{ }_{-\gamma}-$ Strahlung induzierten Leitfähigkeit von $\mathrm{n}$-Hexan in einer Parallelplatten-Ionisationskammer. Außer der Art und Wirksamkeit der Rekombinationsmechanismen und den sich daraus ergebenden Folgerungen über die räumliche Ionenverteilung in bestrahlten Flüssigkeiten interessierte bei der Durchführung dieser Untersuchung auch die praktische Anwendbarkeit flüssigkeitsgefüllter Ionisationskammern zur direkten Dosisbestimmung in Flüssigkeiten.

\section{Theoretische Betrachtungen}

\subsection{Primärrekombination}

Die beim Ionisationsproze $ß$ auf das freie Elektron übertragene Energie wird durch Stoßprozesse abgebaut. Entscheidend für die Wahrscheinlichkeit der Primärrekombination des Elektrons mit seinem positiven Mutterion ist der Abstand $R$ des Elektrons vom Mutterion zum Zeitpunkt seiner Thermalisierung bzw. seines Einfangs durch neutrale Moleküle. Als kritischer Abstand $R_{\mathrm{k}}$ kann daher der Abstand

12 H. A. Kramers, Physica 18, 665 [1952].

13 D. E. Lea, Proc. Cambridge Phil. Soc. 30, 80 [1934].

11 S. Lenketr u. H. G. Ebert, Naturwiss. 51, 237 [1964]. bezeichnet werden, bei dem die Coulomв-Bindungsenergie zwischen den beiden entgegengesetzt geladenen Ladungsträgern gleich der thermischen Energie $k T$ ist. Dabei kann als Ladung der Rekombinationspartner eine Elementarladung $e$ angenommen werden. Setzt man als kritischen Abstand

$$
R_{\mathrm{k}}=e^{2} /(\varepsilon k T)
$$

ein $(\varepsilon=$ Dielektrizitätskonstante der Flüssigkeit), so folgt aus der Theorie von OnSAger ${ }^{14}$ für die Wahrscheinlichkeit $\varphi_{\mathrm{po}}, \mathrm{da} \beta$ ein Elektron im Abstand $R$ bei Abwesenheit eines äußeren elektrischen Feldes der Primärrekombination entgeht:

$$
\varphi_{\mathrm{po}}=e^{-R_{\mathrm{k}} / R} .
$$

Im Falle von n-Hexan ergibt sich bei $20^{\circ} \mathrm{C} \quad(\varepsilon$ $=1,890)$ ein kritischer Abstand $R_{\mathrm{k}}=300 \AA$. Die meisten Elektronen, die beim Ionisationsprozeß gebildet werden, haben in Flüssigkeiten eine Reichweite $R \ll R_{\mathrm{k}}$, so daß sie sofort nach ihrer Entstehung wieder mit ihrem positiven Mutterion rekombinieren. Die mittlere Zeitdauer dieses primären Rekombinationsprozesses in Flüssigkeiten ist kleiner als $10^{-12} \mathrm{sec}$, wenn man die Elektronen als frei beweglich annimmt ${ }^{15}$. Wenn das Elektron jedoch bereits in gebundener bzw. solvatisierter Form vorliegt, ist die Zeitdauer der Primärrekombination wesentlich größer $\left(\sim 10^{-9} \mathrm{sec}\right)$.

Bei Anlegen eines äußeren elektrischen Feldes hängt die Entkommwahrscheinlichkeit vom Betrag $E$ der Feldstärke und dem Winkel zwischen der Feldrichtung und der Verbindungslinie der beiden Rekombinationspartner ab. Für $\delta$-Strahlen kann eine Gleichverteilung über alle Winkel angenommen werden. Unter dieser Voraussetzung nimmt die Entkommwahrscheinlichkeit $\varphi_{\mathrm{p}}$ mit der Feldstärke entsprechend einer Beziehung

$$
\varphi_{\mathrm{p}}(R, E)=\varphi_{\mathrm{po}}(R)[1+f(R, E)]
$$

zu. Die Funktion $f(R, E)$ wurde von Onsager ${ }^{14}$ berechnet und kann nach F REEMAN $^{7}$ für Feldstärken $E<70 \mathrm{kV} / \mathrm{cm}$ durch die Reihe

$$
\begin{aligned}
f(R, E)= & a E R_{\mathrm{k}}+a^{2} E^{2}\left(0,375 R_{\mathrm{k}}^{2}-0,750 R_{\mathrm{k}} R\right) \\
& +a^{3} E^{3}\left(0,0695 R_{\mathrm{k}}^{3}-0,417 R_{\mathrm{k}}^{2} R\right. \\
& \left.+0,417 R_{\mathrm{k}} R^{2}\right) \\
& +a^{4} E^{4}\left(0,0076 R_{\mathrm{k}}^{4}-0,091 R_{\mathrm{k}}^{3} R\right. \\
& \left.+0,274 R_{\mathrm{k}}^{2} R^{2}-0,183 R_{\mathrm{k}} R^{3}\right)
\end{aligned}
$$

14 L. Onsager, Phys. Rev. 54,554 [1938].

15 A. H. Samuel u. J. L. Magee, J. Chem. Phys. 21, 1080 [1953]. 
dargestellt werden. Dabei ist $a=e / 2 k T\left(a=19,8 V^{-1}\right.$ bei $20^{\circ} \mathrm{C}$ ), $R$ und $R_{\mathrm{k}}$ in $\mathrm{cm}$ und $E$ in $\mathrm{V} / \mathrm{cm}$ einzusetzen.

In Abb. 1 ist die Funktion $1+f(R, E)=\varphi_{\mathrm{p}} / \varphi_{\mathrm{po}}$ für n-Hexan bei $20^{\circ} \mathrm{C}$ in Abhängigkeit von der Feldstärke und für verschiedene Werte des Abstandes $R$ aufgetragen. Ferner ist $\varphi_{\text {po }}$ nach Gl. (2) angegeben. In dem hier vor allem interessierenden Be-

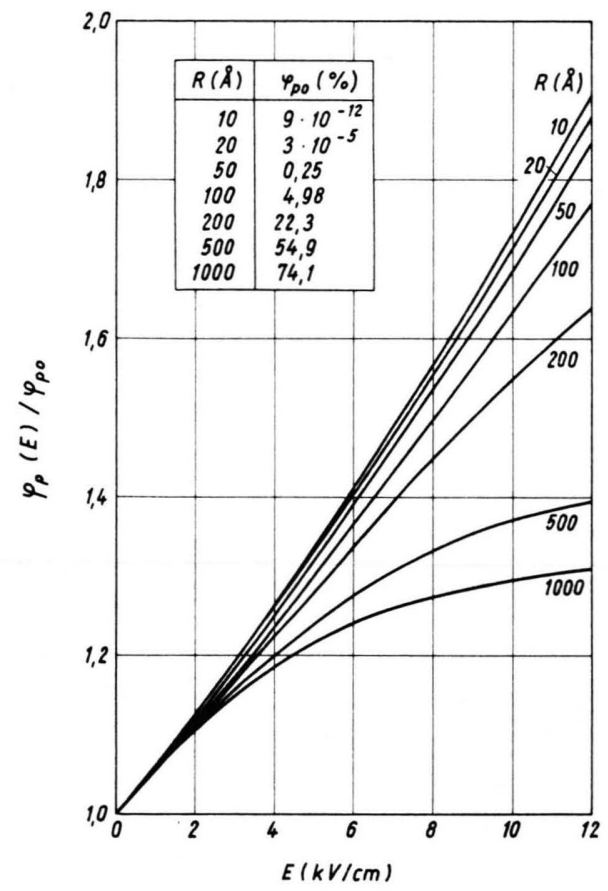

Abb. 1. Entkommwahrscheinlichkeit $\varphi_{\mathrm{p}}$ für die Primärrekombination in $\mathrm{n}$-Hexan $\left(20^{\circ} \mathrm{C}\right.$; kritischer Abstand $\left.R_{\mathrm{k}}=300 \AA\right)$ in Abhängigkeit von der Feldstärke $E$; berechnet für verschiedene Anfangsabstände $R$ der Ionen eines Ionenpaares.

reich $R<R_{\mathrm{k}}$ nimmt die Entkommwahrscheinlichkeit bei Feldstärken $\leqq 10 \mathrm{kV} / \mathrm{cm}$ annähernd linear mit der Feldstärke zu, so daß in diesem Fall $f(R, E)=g(R) \cdot E$ gesetzt werden kann, wobei $g$ nur vom Abstand $R$ abhängt. Somit ergibt sich die Möglichkeit, aus der Feldstärkenabhängigkeit der Primärrekombination Aufschluß über den Anfangsabstand $R$ der Ionen, d.h. über die Reichweite der Elektronen in der Flüssigkeit zu erhalten.

Bei der praktischen Anwendung der Gln. (2) und (3) ist zu berücksichtigen, daß in Wirklichkeit eine Reichweitenverteilung vorliegt. Einerseits haben die beim Ionisationsprozeß ausgelösten Elektronen verschiedene Anfangsenergien, andererseits haben aber auch Elektronen gleicher Anfangsenergie eine Reichweitenverteilung, die sich von 0 bis zu einem Maximalwert erstreckt, der der Bahnlänge dieser Elektronen entspricht. Sei $v(R) \mathrm{d} R$ der relative Anteil der Elektronen mit einer Reichweite im Intervall $R$ bis $R+\mathrm{d} R$, so beträgt die mittlere Entkommwahr. scheinlichkeit bei $E=0$ :

$$
\bar{\varphi}_{\mathrm{po}}=\int_{0}^{\infty} \boldsymbol{v}(R) \cdot \varphi_{\mathrm{po}}(R) \mathrm{d} R .
$$

Aus Messungen der Leitfähigkeit kann ferner das Verhältnis

$$
\overline{\varphi_{\mathrm{p}} / \varphi_{\mathrm{p} 0}}=1+\int_{0}^{\infty} f(R, E) \cdot \nu(R) \mathrm{d} R=1+\bar{f}(E)
$$

ermittelt werden, dessen Abhängigkeit von der Feldstärke Aufschluß über den mittleren, für die Primärrekombination effektiven Abstand $R_{\text {eff }}$ der Elektronen von ihren Mutterionen gibt. Der Vorgang der Primärrekombination - der bei Gasen normaler Dichte vernachlässigbar ist - reduziert die strahleninduzierte Leitfähigkeit in Flüssigkeiten erheblich. Die primäre Ionisationsrate in der Flüssigkeit, d. h. die Zahl der pro Zeit- und Volumeneinheit durch die Strahlung gebildeten Ionenpaare, beträgt

$$
I_{\mathrm{p}}=(\dot{D} \varrho) / W
$$

wobei $\dot{D}$ die Dosisleistung, $\varrho$ die Flüssigkeitsdichte $\left(\varrho=0,66 \mathrm{~g} / \mathrm{cm}^{3}\right.$ für $\mathrm{n}-\mathrm{Hexan}$ bei $\left.20{ }^{\circ} \mathrm{C}\right)$ und $W$ der mittlere Energieverlust der Strahlung pro gebildetem Ionenpaar ist. Nach Abzug des durch Primärrekombination verlorengehenden Anteils folgt somit für die effektive Ionisationsrate, die für die strahleninduzierte Leitfähigkeit in der Flüssigkeit maßgebend ist:

$$
I=\bar{\varphi}_{\mathrm{p}} I_{\mathrm{p}}=\varrho \dot{D} \varphi_{\mathrm{p} 0}(1+f) / W .
$$

Da $f$ von der Feldstärke abhängt, nimmt somit die effektive Ionisationsrate gemäß Abb. 1 mit steigender Feldstärke zu. Das in Gl. (8) eingehende Verhältnis $\bar{\varphi}_{\mathrm{p}} / W$ ist mit dem $G$-Wert für die der Primärrekombination entgehenden Ionenpaare durch die Beziehung $G_{\mathrm{p}}=100 \cdot \bar{\varphi}_{\mathrm{p}} / W(\mathrm{eV})$ verknüpft.

Eine direkte Messung des mittleren Energieverlustes $W$ pro primär gebildetem Ionenpaar wie in Gasen ist in Flüssigkeiten nicht möglich, da bei den erreichbaren Feldstärken in dielektrischen Flüssigkeiten stets $\varphi_{\mathrm{p}} \ll 1$ ist. Bei gasförmigen gesättigten Kohlenwasserstoffen liegt $W$ im Bereich von 20 bis 
$30 \mathrm{eV}^{16-20}$. Da bei gleicher Zusammensetzung das Massenbremsvermögen von Flüssigkeiten und Gasen für Elektronen sich nur durch den Dichteeffekt unterscheidet und der Dichteeffekt bei Elektronenenergien unterhalb $1 \mathrm{MeV}$ vernachlässigbar ist, ist $\mathrm{zu} \mathrm{er}$ warten, daß der mittlere Energieverlust $W$ pro primär gebildetem Ionenpaar in flüssigem Hexan ebenfalls im Bereich von $20-30 \mathrm{eV}$ liegt. Dafür sprechen auch die Ergebnisse strahlenchemischer Untersuchungen über die Ausbeute von $\mathrm{H}_{2}$ und organischen Produkten bei der Radiolyse von gasförmigem und flüssigem Hexan ${ }^{21}$.

\subsection{Sekundärrekombination}

Nach Ablauf der primären Rekombinationsphase setzt die sekundäre Rekombination (Volumenrekombination) ein, die dem Rekombinationsgesetz

$$
\mathrm{d} n_{ \pm} / \mathrm{d} t=-K n_{+} n_{-}
$$

gehorcht. Dabei ist $K$ der Ionen-Rekombinationskoeffizient und $n_{+}$bzw. $n_{-}$die Konzentration der positiven bzw. negativen Ionen. Bei einem homogen bestrahlten Flüssigkeitsvolumen $V$ folgt somit die spezifische Stromstärke (Stromstärke/V) zu

wobei

$$
i=\varphi_{\mathrm{s}} e I,
$$

$$
\varphi_{\mathrm{s}}=1-\frac{K}{I V} \int_{V} n_{+} n_{-} \mathrm{d} V
$$

den relativen Anteil der Ionen angibt, die der Sekundärrekombination entgehen.

Bei den hier beschriebenen Untersuchungen wurde eine Parallelplatten-Ionisationskammer verwendet. Da Raumladungseffekte vernachlässigt werden können, steigt $n_{+}$vom Wert 0 an der Anode linear mit dem Abstand $x$ von der Anode an; entsprechendes gilt in umgekehrter Richtung für $n_{-}$. Sei $d$ der Elektrodenabstand und $u$ die Beweglichkeit der positiven bzw. negativen Ionen, so ergibt sich aus der Ionenbilanz:

$$
\begin{aligned}
& n_{+}(x)=\frac{\varphi_{\mathrm{s}} I}{u_{+} E} x, \\
& n_{-}(x)=\frac{\varphi_{\mathrm{B}} I}{u_{-} E}(d-x) .
\end{aligned}
$$

16 W. P. Jesse u. J. Sadauskis, Phys. Rev. 90, 1120 [1953].

17 T. E. Bortner u. G. S. Hurst, Phys. Rev. 93, 1236 [1954].

18 P. Huber, Nucl. Sci. Ser. Rep. No. 29 [1960], 123-133.

19 J. Booz u. H. G. Ebert, Strahlentherapie 120, 7 [1963].

20 T. D. Strickler, J. Chem. Phys. 67, 825 [1963].

21 J. H. Futrell, J. Am. Chem. Soc. 81, 5921 [1959].

22 O. H. Le Blanc, J. Chem. Phys. 30, 1443 [1959].
Durch Einsetzen dieser Gleichungen in Gl. (11) und Integration folgt für $\varphi_{\mathrm{s}}$ die quadratische Gleichung

$$
\varphi_{\mathrm{s}}=1-\frac{K I d^{2}}{6 u_{+} u_{-} E^{2}} \cdot \varphi_{\mathrm{s}}^{2}
$$

mit der Lösung:

$$
\varphi_{\mathrm{s}}=\frac{2}{1+\sqrt{1+2} K d^{2} I /\left(3 u_{+} u_{-} E^{2}\right)} .
$$

Dies ist die Gleichung einer Sättigungsfunktion, die mit zunehmender Feldstärke gegen 1 geht.

In dem hier zur Diskussion stehenden Fall der Ionenrekombination in Flüssigkeiten geringer Ionenkonzentration ist die Langevinsche Theorie der Rekombination anwendbar, wonach der Rekombinationskoeffizient mit den Ionenbeweglichkeiten durch die Beziehung

$$
K=4 \pi e^{2}\left(u_{+}+u_{-}\right) / \varepsilon
$$

verknüpft ist. Für $\mathrm{n}$-Hexan bei $20{ }^{\circ} \mathrm{C}$ ist somit das in Gl. (14) eingehende Verhältnis $K / u_{+} u_{-}=1,0$. $10^{-6}\left(u_{+}+u_{-}\right) / u_{+} u_{-}$, wobei $K$ in $\mathrm{cm}^{3} / \mathrm{sec}$ und $u$ in $\mathrm{cm}^{2} / \mathrm{V}$ sec einzusetzen sind.

Über die Ionenbeweglichkeiten in n-Hexan liegen zahlreiche Messungen vor ${ }^{22-28}$. Für positive Ionen ergibt sich bei $20^{\circ} \mathrm{C}$ übereinstimmend ein Mittelwert von $u_{+}=4,5 \cdot 10^{-4} \mathrm{~cm}^{2} / \mathrm{V} \mathrm{sec}$ (Fehler ca. $\pm 10 \%)$. Demgegenüber streuen die Ergebnisse für negative Ionen erheblich und umfassen den Bereich $u_{-}=2 \cdot 10^{-4}-10 \cdot 10^{-4} \mathrm{~cm}^{2} / \mathrm{V}$ sec. Daraus geht hervor, daß die negativen Ladungsträger keine freien Elektronen sind. Die wahrscheinliche Ursache für die Schwankungen der negativen Ionenbeweglichkeit sind Verunreinigungen im Hexan, insbesondere Sauerstoffspuren, die eine hohe Elektronenaffinität besitzen. Als sicherster Wert für reines $n$-Hexan ohne Sauerstoff kann $u_{-}=9 \cdot 10^{-4} \mathrm{~cm}^{2} / \mathrm{V} \mathrm{sec}\left(20^{\circ} \mathrm{C}\right)$ angesehen werden. Mit $u_{+}=4,5 \cdot 10^{-4} \mathrm{~cm}^{2} / \mathrm{V} \mathrm{sec}$ und $u_{-}=9 \cdot 10^{-4} \mathrm{~cm}^{2} / \mathrm{V}$ sec folgt aus Gl. (15) ein Rekombinationskoeffizient von $K=1,3 \cdot 10^{-9} \mathrm{~cm}^{3} / \mathrm{sec}$ und ein Verhältnis $K / u_{+} u_{-}=3,3 \cdot 10^{-3} V^{2} \mathrm{sec} / \mathrm{cm}$.

\subsection{Theoretischer Verlauf der Strom-Feldstärke- Charakteristik}

Unter Berücksichtigung der Entkommwahrscheinlichkeiten $\varphi_{\mathrm{p}}$ bzw. $\varphi_{\mathrm{s}}$ für die Primär- bzw. Sekundär-

${ }^{23}$ M. J. Morant, Nature 187, 48 [1960].

24 P. Chong u. Y. Inuishi, Technol. Rep. Osaka Univ. 10, 545 [1960].

25 I. Adamczewski, Atompraxis 7, 327 [1961].

26 D. W. Swan, Nature 190, 904 [1961].

27 O. Gzowski, Z. Phys. Chem. 221, 288 [1962].

28 E. GAZDA, Nature 200, 767 [1963]. 
rekombination in einer dielektrischen Flüssigkeit folgt für die strahleninduzierte spezifische Stromstärke:

$$
i=e \varphi_{\mathrm{s}} I=e \bar{\varphi}_{\mathrm{p}} \varphi_{\mathrm{s}} I_{\mathrm{p}} .
$$

Bei einer Parallelplatten-Ionisationskammer ist somit gemäß den Gln. (8) und (14) eine Strom-Feldstärke-Charakteristik zu erwarten, deren Verlauf durch die Beziehung

$i(E)=i_{\mathrm{p}}(E) \cdot \frac{2}{1+\sqrt{1+\left(2 K d^{2} i_{\mathrm{p}}(E) / 3 u_{+} u_{-} e E^{2}\right)}}$

beschrieben wird. Dabei gibt

$$
i_{\mathrm{p}}(E)=e \varrho \dot{D} \bar{\varphi}_{\mathrm{po}}[1+f(E)] / W
$$

die spezifische Stromstärke an, die ohne Sekundärrekombination erreicht wurde.

In Abb. 2 ist die aus den Gln. (17) und (18) unter Berücksichtigung der Gln. (1), (2) und (4) berechnete Abhängigkeit des Verhältnisses $i / \dot{D}$ von der Feldstärke bei verschiedenen Dosisleistungen in n-Hexan aufgetragen. Der Berechnung wurden die in der Abb. 2 eingetragenen Ionenbeweglichkeiten

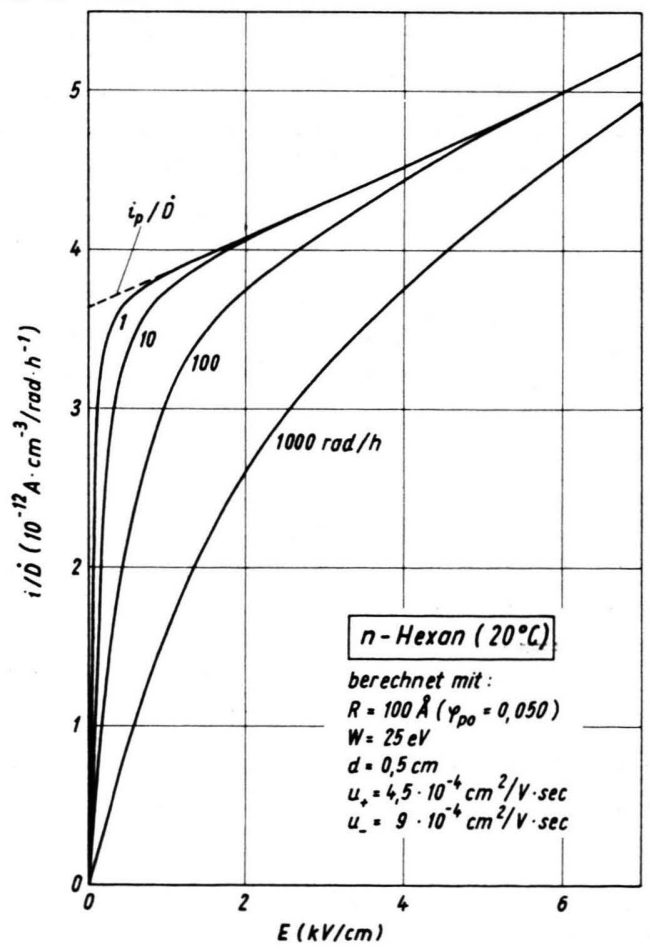

Abb. 2. Theoretisch zu erwartende Feldstärkeabhängigkeit der durch $\gamma$-Strahlung induzierten, spezifischen Stromstärke $i$ pro $\mathrm{rad} / \mathrm{h}$ in einer mit $\mathrm{n}$-Hexan gefüllten ParallelplattenIonisationskammer (Parameter: Dosisleistung $\dot{D}$ ). in reinem Hexan bei $20^{\circ} \mathrm{C}$ und ein Elektrodenabstand von $5 \mathrm{~mm}$ zugrunde gelegt. Ferner wurde $W=25 \mathrm{eV}$ und eine mittlere effektive Reichweite $R_{\text {eff }}=100 \AA$ der Elektronen angenommen; letzteres bedeutet nach Abb. 1, daß ohne äußeres Feld 5\% der durch die Strahlung erzeugten Ionenpaare der Primärrekombination entgehen.

Abb. 2 läßt den Einfluß von Primär- und Sekundärrekombination klar erkennen. Der starke Anstieg der $i / \dot{D}$-Kurve bei geringen Feldstärken wird bedingt durch die abnehmende Sekundärrekombination und ist abhängig von der Dosisleistung. Mit zunehmender Feldstärke geht $\varphi_{\mathrm{s}} \rightarrow 1$, d.h. es tritt Sättigung in Bezug auf die Sekundärrekombination ein; bei einer Dosisleistung von $1 \mathrm{rad} / \mathrm{h}$ wird diese Sättigung praktisch bereits bei Feldstärken oberhalb etwa $0,5 \mathrm{kV} / \mathrm{cm}$ erreicht, während bei $100 \mathrm{rad} / \mathrm{h}$ etwa $4 \mathrm{kV} / \mathrm{cm}$ notwendig sind. In diesem Sättigungsbereich fällt die $i / \dot{D}$-Kurve mit der durch Gl. (18) definierten $i_{\mathrm{p}} / \dot{D}$-Kurve zusammen, so daß der weitere langsame, annähernd linear mit der Feldstärke erfolgende Anstieg der Stromstärke allein durch die Zunahme der Entkommwahrscheinlichkeit $\bar{\varphi}_{\mathrm{p}}$ bei der Primärrekombination bestimmt wird. $\mathrm{Da} \bar{\varphi}_{\mathrm{p}}$ unabhängig von der Dosisleistung ist, ist in diesem Bereich auch das Verhältnis $i / \dot{D}$ unabhängig von der Dosisleistung.

Betrachtet man bei konstanter Feldstärke die Abhängigkeit der Stromstärke von der Dosisleistung, so ist bei niedriger Dosisleistung $i \sim \dot{D}$. Mit zunehmender Dosisleistung macht sich der Einfluß der Sekundärrekombination bemerkbar; aus Gl. (17) folgt, daß bei hohen Dosisleistungen schließlich eine Abhängigkeit $i \sim \sqrt{\dot{D}}$ erreicht wird.

\section{Meßanordnung}

\subsection{Ionisationskammer}

Zur Leitfähigkeitsmessung wurde eine Parallelplatten-Ionisationskammer verwendet, deren Wand aus Teflon bestand und mit Schliffansätzen zum Einfüllen und Ablassen des Hexans versehen war (Abb. 3). Die kreisförmige Meßelektrode hatte einen Durchmesser von $40 \mathrm{~mm}$ und war von einem $9 \mathrm{~mm}$ breiten Schutzring umgeben, um ein homogenes elektrisches Feld im Meßvolumen zu gewährleisten. Die ihr gegenüberstehende Hochspannungselektrode hatte einen Durchmesser von $50 \mathrm{~mm}$ und konnte mit Hilfe einer Mikrometerschraube verschoben werden, so daß der Elektrodenabstand von $0-5 \mathrm{~mm}$ variiert werden konnte. Die Elektroden bestanden aus Aluminium, um Elektronen- 


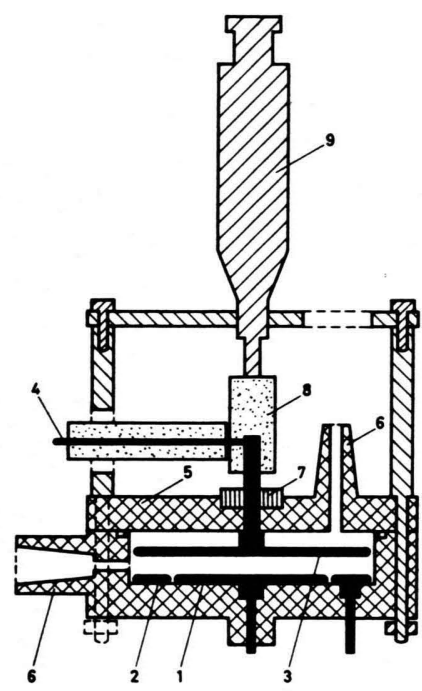

Abb. 3. Querschnitt durch die Ionisationskammer, Typ 1. 1 Meßelektrode, 2 Schutzring, 3 Hochspannungselektrode, 4 Hochspannungszuführung, 5 Kammerwand aus Teflon, 6 Einfüllstutzen, 7 Hutmanschette, 8 Plexiglasisolation, 9 Mikrometerschraube.

gleichgewicht zwischen Elektroden und der Füllflüssigkeit (n-Hexan) zu erreichen.

Der über die Meßelektrode abfließende Strom wurde mit einem Elektrometer (K e ithle y Modell 610 A) mit einem Eingangswiderstand größer als $10^{14} \Omega$ gemessen und mit einem Linienschreiber registriert. Die Genauigkeit der Strommessung betrug $4 \%$ des Vollausschlags des Instruments im Meßbereich von $10^{-8}$ bis $10^{-13} \mathrm{~A}$. Die an der oberen Elektrode angelegte positive Spannung konnte im Bereich von $50-3000 \mathrm{~V}$ variiert werden.

\subsection{Hexan-Füllung und Eigenleitfähigkeit}

Vergleichsmessungen bei $\mathrm{N}_{2^{-}}$und Hexanfüllung der Ionisationskammer zeigten, daß die Grundleitfähigkeit bei fehlender äußerer Bestrahlung im wesentlichen durch die Eigenleitfähigkeit des Hexans bestimmt wurde und demgegenüber der Isolationsstrom über die Kammerwand vernachlässigbar war. Die Eigenleitfähigkeit des Hexans hängt stark vom Reinheitsgrad ab. Messungen mit handelsüblichem, chemisch reinem oder chromatographisch reinem n-Hexan ergaben eine Eigenleitfähigkeit von $10^{-16} \Omega^{-1} \mathrm{~cm}^{-1}$; bei $\mathrm{n}$-Hexan für spektroskopische Zwecke (Hersteller: Fa. M e rck) wurde eine Eigenleitfähigkeit von $10^{-17} \Omega^{-1} \mathrm{~cm}^{-1}$ gemessen. Durch extreme Nachreinigung konnte zwar die Eigenleitfähigkeit noch um 1-2 Zehnerpotenzen erniedrigt werden, jedoch wurde dann eine mangelnde Reproduzierbarkeit der Meßwerte festgestellt. Die vorliegenden Untersuchungen wurden daher unter Verzicht auf eine besondere Nachreinigung mit spektrographisch reinem $\mathrm{n}$-Hexan durchgeführt. Der Wassergehalt dieses n-Hexans, der für seine Eigenleitfähigkeit von wesentlicher Bedeutung ist, wurde mit Hilfe eines Massen- spektrometers geprüft und lag unter der Nachweisgrenze von $0,01 \%$.

Das n-Hexan wurde in die sorgfältig gereinigte und mit $\mathrm{N}_{2}$ gespülte Ionisationskammer eingefüllt, ohne dabei mit der Außenluft in Berührung zu kommen. Vor Bestrahlung wurde das n-Hexan in der Kammer einer mehrstündigen elektrischen Nachreinigung unterworfen. Die Leitfähigkeitsmessungen wurden bei einer Temperatur von $(20 \pm 2){ }^{\circ} \mathrm{C}$ durchgeführt, so daß der Einfluß von Temperaturschwankungen auf die Leitfähigkeit unberücksichtigt bleiben konnte.

\subsection{Bestrahlungsanordnung und Dosisbestimmung}

Die hexangefüllte Ionisationskammer wurde senkrecht zur Feldrichtung mit $\mathrm{Co}^{60}-\gamma$-Strahlen bestrahlt. Die Dosisleistung in Luft am Ort der flüssigkeitsgefüllten Ionisationskammer wurde mit Hilfe von luftäquivalenten Fingerhutionisationskammern (Hersteller: Fa. Vi c t o r e e n, USA) gemessen und unter Berücksichtigung der Schwächung in der Wand und im Hexan der Kammer umgerechnet auf die Dosisleistung in Hexan im Zentrum des Meßvolumens. Der Einfluß der Wandschwächung wurde durch Vergleichsmessungen mit Kondensatorionisationskammern, die in das Meßvolumen der Flüssigkeitsionisationskammer eingebracht wurden, kontrolliert. Danach dürfte der Fehler bei den angegebenen Dosiswerten im Hexan maximal $\pm 10 \%$ betragen.

\section{Versuchsergebnisse und Diskussion}

\subsection{Strom-Feldstärke-Charakteristik}

Die Strom-Feldstärke-Charakteristik der Hexangefüllten Ionisationskammer wurde bei Dosisleistungen im Bereich von $0,1-150 \mathrm{rad} / \mathrm{h}$ und bei Elektrodenabständen von $0,1-0,5 \mathrm{~cm}$ aufgenommen. Als typische Beispiele sind in Abb. 4 die gemessenen Kurven bei 4,5 und $150 \mathrm{rad} / \mathrm{h}$ und verschiedenen Elektrodenabständen aufgetragen. Dabei ist als Ordinate das Verhältnis der spezifischen Stromstärke $i$ zur Dosisleistung $\dot{D}$ nach Abzug des durch die Eigenleitfähigkeit bedingten Stromanteils angegeben.

Der Verlauf der Strom-Feldstärke-Charakteristik stimmt mit dem nach Gl. (17) theoretisch zu erwartenden Verlauf überein (vgl. auch Abb. 2). Bei einer Dosisleistung von 4,5 bzw. $150 \mathrm{rad} / \mathrm{h}$ wird Sättigung in bezug auf die Sekundärrekombination $\left(\varphi_{\mathrm{s}}=1\right)$ praktisch bei Feldstärken oberhalb 1 bzw. $3 \mathrm{kV} / \mathrm{cm}$ erreicht. In diesem Bereich steigt die Stromstärke langsam proportional mit der Feldstärke an, wie es auf Grund der Onsagerschen Theorie der Primärrekombination zu erwarten ist, wenn die mittlere Reichweite $R$ der Elektronen kleiner ist als der kritische Abstand $R_{\mathrm{k}}=300 \AA$ in 

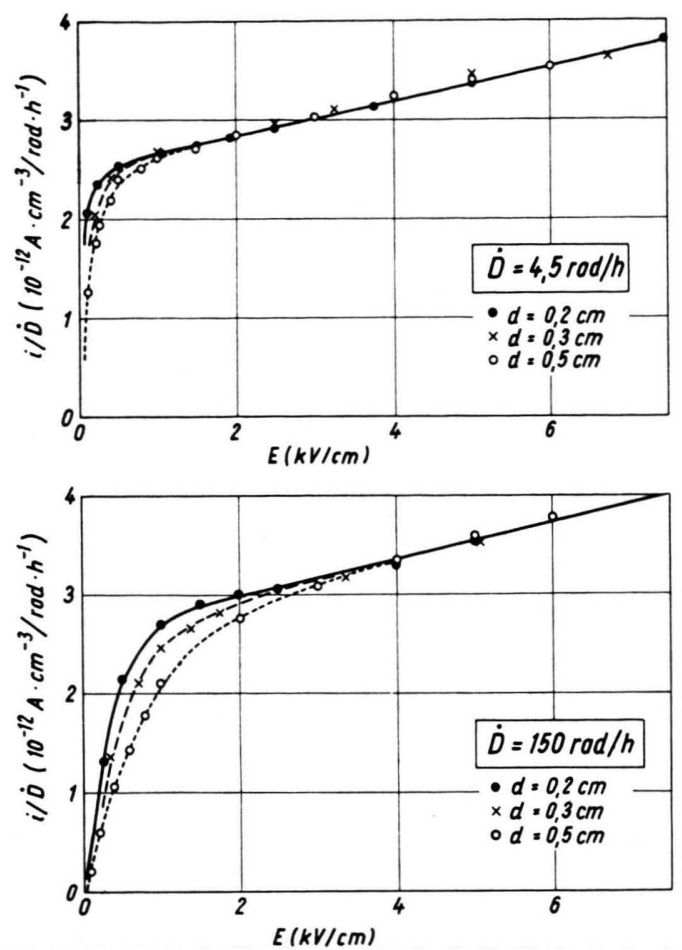

Abb. 4. Gemessene Strom-Feldstärke-Charakteristik einer mit n-Hexan gefüllten Parallelplatten-Ionisationskammer bei Einwirkung von $\mathrm{Co}^{60}-\gamma$-Strahlung (Parameter: Dosisleistung $\dot{D}$ und Elektrodenabstand $d$ ).

Hexan (vgl. Abb. 1). Ferner ist dann in Einklang mit der Theorie das Verhältnis $i / \dot{D}$ innerhalb der Fehlergrenzen der Messungen unabhängig von der Dosisleistung und dem Elektrodenabstand.

\subsection{Entkommwahrscheinlichkeit bei der Primär- rekombination}

Der lineare Bereich der Strom-Feldstärke-Charakteristik gibt nach Gl. (18) und Abb. 2 die Stromstärke $i_{\mathrm{p}}$ an, die ohne Sekundärrekombination erreicht wird. Durch lineare Extrapolation gegen $E=0$ folgt der Wert für $i_{\mathrm{po}}=e \varrho D \cdot \varphi_{\mathrm{po}} / W$, aus dem das Verhältnis $\varphi_{\mathrm{po}} / W$ bzw. der Wert von $G_{\mathrm{po}}$ für die Primärrekombination ohne äußeres Feld berechnet werden kann. Die auf diese Weise aus Meßreihen bei verschiedener Dosisleistung und Elektrodenabstand resultierenden Werte für $i_{\mathrm{po}}$ und $\varphi_{\mathrm{po}} / W$ sind in Tab. 1 angegeben. Daraus ergibt sich als Mittelwert und Streuung

$$
\varphi_{\mathrm{po}} / W=(0,0013 \pm 0,0001) \mathrm{eV}^{-1},
$$

was einem Wert von $G_{\mathrm{p} 0}=0,13$ entspricht. Dieser Wert stimmt innerhalb der Unsicherheitsgrenzen mit dem Wert $G_{\mathrm{po}}=0,10 \pm 0,05$ für $\mathrm{Co}^{60}{ }_{-\gamma}-$ Strahlen überein, der von Freeman ${ }^{7,8}$ sowie Allen und Hummel $^{3}$ in anderer Weise abgeleitet wurde. Das hier beschriebene Bestimmungsverfahren hat den Vorteil, daß es unabhängig von der Ionenbeweglichkeit ist und die Extrapolation gegen $E=0$ sehr genau durchführbar ist. Die Streuung der Werte für $\varphi_{\mathrm{po}} / W$ in Tab. 1 liegt innerhalb der Fehlergrenzen der Messung. Der systematische Fehler wird in erster Linie durch die Dosisbestimmung und die Strommessung verursacht und dürfte $10-20 \%$ nicht überschreiten.

Die Unabhängigkeit der Entkommwahrscheinlichkeit $\varphi_{\mathrm{po}}$ von der Dosisleistung bestätigt die theoreti-

\begin{tabular}{|c|c|c|c|c|c|c|}
\hline$\dot{D}_{\text {Hexan }} 1$ & $\begin{array}{l}d^{2} \\
\mathrm{~cm}\end{array}$ & $\stackrel{i_{\mathrm{p} 0}}{10^{-10} \mathrm{~A} / \mathrm{cm}^{3}}$ & $\begin{array}{c}\overline{\varphi_{\mathrm{p} 0}} / W \\
10^{-3} \mathrm{eV}^{-1}\end{array}$ & $\begin{array}{c}\mathrm{d} i_{\mathrm{p}} / \mathrm{d} E \\
10^{-14} \mathrm{~A} / \mathrm{Vcm}{ }^{2}\end{array}$ & $\begin{array}{c}\bar{g} \\
\mathrm{~cm} / \mathrm{kV}\end{array}$ & $\begin{array}{c}\frac{u_{+}+u_{-}}{u_{+} u_{-}} \\
10^{3} \mathrm{Vsec} / \mathrm{cm}^{2}\end{array}$ \\
\hline $\begin{array}{r}1,5 \\
1,8 \\
2,0 \\
2,5 \\
3,3 \\
4,5 \\
4,5 \\
4,5 \\
4,5 \\
4,5 \\
100 \\
150 \\
150 \\
150 \\
150 \\
150\end{array}$ & $\begin{array}{l}0,5 \\
0,5 \\
0,5 \\
0,5 \\
0,5 \\
0,5 \\
0,4 \\
0,3 \\
0,2 \\
0,1 \\
0,5 \\
0,5 \\
0,4 \\
0,3 \\
0,2 \\
0,1\end{array}$ & $\begin{array}{l}0,036 \\
0,043 \\
0,050 \\
0,065 \\
0,082 \\
0,110 \\
0,115 \\
0,112 \\
0,113 \\
0,107 \\
2,70 \\
3,65 \\
3,40 \\
3,65 \\
3,90 \\
3,5\end{array}$ & $\begin{array}{l}1,32 \\
1,29 \\
1,37 \\
1,40 \\
1,35 \\
1,33 \\
1,39 \\
1,36 \\
1,35 \\
1,30 \\
1,46 \\
1,31 \\
1,24 \\
1,32 \\
1,42 \\
1,26\end{array}$ & $\begin{array}{l}0,021 \\
0,027 \\
0,032 \\
0,040 \\
0,052 \\
0,073 \\
0,081 \\
0,081 \\
0,076 \\
0,077 \\
2,32 \\
3,30 \\
4,20 \\
2,63 \\
2,80 \\
2,5\end{array}$ & $\begin{array}{l}0,056 \\
0,063 \\
0,062 \\
0,060 \\
0,063 \\
0,066 \\
0,070 \\
0,072 \\
0,068 \\
0,071 \\
0,086 \\
0,088 \\
0,12 \\
0,071 \\
0,075 \\
0,07\end{array}$ & $\begin{array}{l}7 \\
6 \\
5 \\
7 \\
7 \\
6 \\
7 \\
- \\
- \\
-6 \\
4,5 \\
3,5 \\
3,5 \\
- \\
-\end{array}$ \\
\hline
\end{tabular}

$11 \mathrm{rad} / \mathrm{h} \triangleq 1,735 \cdot 10^{10} \mathrm{eV} / \mathrm{g} \cdot \mathrm{sec} \quad{ }^{2}$ Das zugehörige Meßvolumen ist $V\left(\mathrm{~cm}^{3}\right)=12,6 \cdot d(\mathrm{~cm})$

Tab. 1. Zusammenstellung der Versuchsergebnisse über die primäre und sekundäre Rekombination in $\mathrm{n}-\mathrm{Hexan}$. 
schen Überlegungen über die Primärrekombination. Setzt man für $\mathrm{n}$-Hexan $W=(25 \pm 5) \mathrm{eV}$ ein (vgl. 1.1), so folgt aus dem gemessenen Mittelwert für $\varphi_{\mathrm{po}} / W$ unter Berücksichtigung der Fehlermöglichkeiten eine Entkommwahrscheinlichkeit in n-Hexan von $\varphi_{\mathrm{po}}=0,03 \pm 0,01$. Dies bedeutet, daß bei niedrigen Feldstärken im Mittel nur 3\% der durch die Strahlung erzeugten Ionenpaare der Primärrekombination entgehen und zur Leitfähigkeit beitragen können. Eine Feldstärke von etwa $15 \mathrm{kV} / \mathrm{cm}$ ist erforderlich, um diesen Anteil zu verdoppeln. Aus diesem Ergebnis folgt nach Gl. (2) mit $R_{\mathrm{k}}=300 \AA$ (vgl. 1.1) eine mittlere effektive Reichweite der Elektronen $R_{\text {eff }}=(80 \pm 10) \AA$ in n-Hexan.

Eine Nachprüfung dieses Ergebnisses ermöglicht die gemessene Abhängigkeit der Primärrekombination von der Feldstärke. In Tab. 1 ist die gemessene Steigung $\mathrm{d} i / \mathrm{d} E=\mathrm{di}_{\mathrm{p}} / \mathrm{d} E$ im linearen Bereich der Charakteristik $\left(\varphi_{\mathrm{s}}=1\right)$ angegeben. In der vorletzten Spalte dieser Tabelle sind die daraus folgenden Werte für $\bar{g}=\mathrm{d} f / \mathrm{d} E$ angegeben [vgl. Gl. (4) und (6) ]. $\bar{g}$ kann auch durch lineare Extrapolation der gemessenen $i_{\mathrm{p}}(E)$-Kurve gegen $i_{\mathrm{p}}=\mathrm{o}$ ermittelt werden, da nach Gl. (18) die dem Schnittpunkt dieser Geraden mit der Abszisse zugeordnete Feldstärke $E_{0}=-1 / \bar{g}$ ist.

Mit Ausnahme der herausfallenden Meßreihe bei $\dot{D}=150 \mathrm{rad} / \mathrm{h}$ und $d=0,4 \mathrm{~cm}$ ist $\bar{g}$ innerhalb der Fehlergrenzen unabhängig von der Dosisleistung und dem Elektrodenabstand und beträgt im Bereich $E \leqq 10 \mathrm{kV} / \mathrm{cm}$ im Mittel

$$
\bar{g}=(0,068 \pm 0,010) \mathrm{cm} / \mathrm{kV} \text {. }
$$

D. h. bei einer Feldstärke von $10 \mathrm{kV} / \mathrm{cm}$ beträgt die Entkommwahrscheinlichkeit etwa das 1,7fache ihres Werts bei der Feldstärke $E=0$. Geht man von dem oben abgeleiteten Wert $R_{\text {eff }}=(80 \pm 10) \AA$ aus, so wäre nach der ONSAGERschen Theorie gemäß Abb. 1 $\bar{g}=0,065 \pm 0,001 \mathrm{~cm} / \mathrm{kV}$ zu erwarten; dieser Wert liegt innerhalb der gemessenen Streubreite.

Zusammenfassend kann daher festgestellt werden, daß der beobachtete Einfluß der Primärrekombination auf die Leitfähigkeit sich mit Hilfe der ONSAGERschen Theorie erklären läßt und sich bei Anwendung dieser Theorie eine mittlere effektive Reichweite der Elektronen beim Ionisationsprozeß in $\mathrm{n}$-Hexan von $(80 \pm 10) \AA$ ergibt. Dies bedeutet, daß beim Ionisationsprozeß in Flüssigkeiten nur die energiereichsten der ausgelösten Elektronen ( $\delta$-Strahlen) eine Chance haben, der Primärrekombination zu entgehen.

\subsection{Volumen-Rekombinationskoeffizient und Ionen- beweglichkeit}

Nach Gl. (14) hängt die Entkommwahrscheinlichkeit $\varphi_{\mathrm{s}}$ von dem Verhältnis $K / u_{+} u_{-}$der beteiligten Ionen ab. Drückt man den Ionen-Rekombinationskoeffizienten $K$ mit Hilfe der Langevinschen Beziehung [Gl. (15)] durch die Ionenbeweglichkeiten aus, so folgt, daß $\varphi_{\mathrm{s}}$ von dem Verhältnis $\left(u_{+}+u_{-}\right) /$ $u_{+} u_{-}$abhängt. Nach Messungen der Ionenbeweglichkeit in $\mathrm{n}$-Hexan ist bei $20^{\circ} \mathrm{C}$ im Mittel $u_{+}=$ $4,5 \cdot 10^{-4} \mathrm{~cm}^{2} / \mathrm{V} \mathrm{sec}$ ( \pm ca. $10 \%$ ), während die Werte für $u_{-}$stark streuen und im Bereich von $2 \cdot 10^{-4}-10 \cdot 10^{-4} \mathrm{~cm}^{2} /$ Vsec liegen (vgl. 1.2). Daraus folgt für das Verhältnis $\left(u_{+}+u_{-}\right) / u_{+} u_{-}$ein Streubereich von $3200-7500 \mathrm{Vsec} / \mathrm{cm}^{2}$; mit dem wahrscheinlich für sehr reines, sauerstofffreies Hexan zutreffenden Wert von $u_{-}=9 \cdot 10^{-4} \mathrm{~cm}^{2} /$ Vsec ergibt sich für dieses Verhältnis ein Wert von $3300 \mathrm{Vsec} /$ $\mathrm{cm}^{2}$.

Aus dem gemessenen Verlauf der Strom-Feldstärke-Charakteristik im unteren Feldstärkebereich (vgl. Abb. 4) läßt sich mit Hilfe von Gl. (17) das Verhältnis $\left(u_{+}+u_{-}\right) / u_{+} u_{-}$direkt bestimmen; die aus den gemessenen Kurven resultierenden Werte sind in der letzten Spalte von Tab. 1 angegeben. Bei einzelnen Meßreihen wurde die Stromstärke bei geringen Feldstärken nicht gemessen, so daß keine Aussage möglich war. Die experimentellen Werte streuen beträchtlich und umfassen den Bereich:

$$
\left(u_{+}+u_{-}\right) / u_{+} u_{-}=3500-7000 \mathrm{Vsec} / \mathrm{cm}^{2} \text {. }
$$

Dieser Bereich deckt sich mit dem oben angegebenen Bereich, der auf Grund der Ionenbeweglichkeiten zu erwarten war. Der gemessene Minimalwert dieses Verhältnisses stimmt überein mit dem für extrem reines Hexan gültigen Wert.

Wie bereits erwähnt, dürften die Schwankungen in erster Linie auf den Einfluß von Spurenstoffen im Hexan zurückzuführen sein, die die Beweglichkeit der negativen Ionen herabsetzen. Dafür spricht auch die aus Tab. 1 erkennbare Tendenz, daß mit zunehmender Dosisleistung das Verhältnis $\left(u_{+}+u_{-}\right) /$ $u_{+} u_{-}$abnimmt und dem Wert für extrem reines Hexan zustrebt. Bei den vorliegenden Untersuchungen handelt es sich wahrscheinlich um geringe Spuren von Sauerstoff, der infolge seiner hohen Elektronenaffinität einen Teil der bei der Primärrekombination entgangenen freien Elektronen sehr schnell an sich reißt und dann durch Anlagerung eines Hexanmoleküls einen $\mathrm{O}_{2}{ }^{-}$-Hexan-Komplex mit relativ ge- 
ringer Ionenbeweglichkeit bildet. Wie aus den im vorigen Abschnitt diskutierten Ergebnissen hervorgeht, haben diese Spuren von Verunreinigungen jedoch keinen nachweisbaren Einfluß auf die Primärrekombination.

\subsection{Dosimetrie mit Hexan-gefüllten Ionisationskammern}

Besonderes Interesse verdient die Frage, inwieweit mit dielektrischen Flüssigkeiten geringer Eigenleitfähigkeit gefüllte Ionisationskammern zur Dosimetrie ionisierender Strahlen geeignet sind. Dabei interessieren die Abhängigkeit der Stromstärke von der Dosisleistung, die untere Nachweisgrenze, die Gewebeäquivalenz von Hexan sowie die Abhängigkeit von der Art und Energie der Strahlung; letztere wird in Abschnitt 4 getrennt behandelt.

Abb. 5 zeigt die strahleninduzierte Stromstärke in Abhängigkeit von der $\gamma$-Dosisleistung in Hexan bei verschiedenen Feldstärken für eine Parallelplattenkammer mit $0,5 \mathrm{~cm}$ Elektrodenabstand. Dabei ist auf der Ordinate das Verhältnis der spezifischen Stromstärke zur Dosisleistung aufgetragen. In Abb. 5 sind neben den Meßwerten die theoretisch berechneten Kurven aufgetragen, die sich aus Gl. (17) mit $\varphi_{\text {po }} / W=0,0013$ und $\bar{g}=0,068 \mathrm{~cm} / \mathrm{kV}$ ergeben (vgl. 3.2). Die experimentellen Werte stimmen innerhalb der Fehlergrenzen mit dem theoretisch zu erwartenden Verlauf überein.

Aus Abb. 5 geht hervor, daß die Stromstärke proportional mit der Dosisleistung ansteigt, solange
Sättigung in bezug auf die Sekundärrekombination vorliegt. Bei einer Feldstärke von $3 \mathrm{kV} / \mathrm{cm}$ erstreckt sich dieser Bereich bereits bis etwa $100 \mathrm{rad} / \mathrm{h}$ und kann durch Erhöhung der Feldstärke weiter ausgedehnt werden. Wie aus der Streuung der Meßwerte in Abb. 5 hervorgeht, dürfte dabei die Genauigkeit der Dosismessung $\pm 5 \%$ betragen. Oberhalb dieses Sättigungsbereichs nimmt $i / \dot{D}$ ab. Bei hohen Werten der Dosisleistung wird nach Gl. (17) $i / \dot{D} \sim 1 / \sqrt{\dot{D}}$, d. h. $i \sim \sqrt{\dot{D}}$, was durch die Messungen von FREEMAN $^{6,7}$ bestätigt wird. Zur Dosimetrie ist es zweckmäßig, bei Sättigung in bezug auf die Sekundärrekombination zu arbeiten, da dann Schwankungen der Ionenbeweglichkeiten infolge von Verunreinigungen keine Rolle spielen.

Als untere Nachweisgrenze kann die strahleninduzierte Stromstärke $i$ angesehen werden, bei der $i$ gleich der durch die Eigenleitfähigkeit $\lambda_{\mathrm{e}}$ bedingten Stromstärke $i_{\mathrm{e}}$ ist. Im Sättigungsbereich für die Volumenrekombination ergibt sich aus den vorliegenden Untersuchungen

$$
\frac{i}{i_{\mathrm{e}}} \approx \frac{2,5 \cdot 10^{-12} d \dot{D}}{\lambda_{\mathrm{e}}}\left(\frac{1}{E}+7 \cdot 10^{-5}\right)
$$

wobei $E$ in $\mathrm{V} / \mathrm{cm}, \dot{D}$ in $\mathrm{rad} / \mathrm{h}, d$ in $\mathrm{cm}$ und $\lambda_{\mathrm{e}}$ in $1 / \Omega \cdot \mathrm{cm}$ einzusetzen sind.

Bei dem hier verwendeten spektroskopisch reinen n-Hexan lag die Eigenleitfähigkeit bei $1 \mathrm{kV} / \mathrm{cm}$ unter $3 \cdot 10^{-17} \Omega^{-1} \mathrm{~cm}^{-1}$. Geht man von diesem ungünstigen Wert aus, so ergibt sich aus Gl. (22) zum Beispiel für einen Elektrodenabstand von

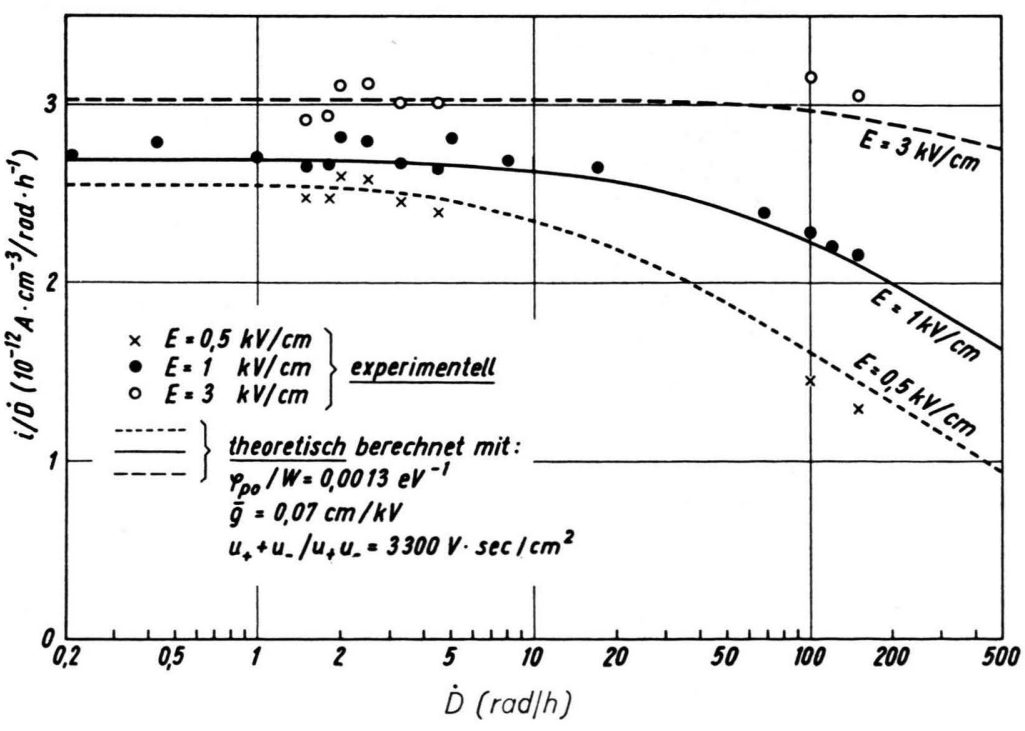

Abb. 5. Durch $\gamma$-Strahlung induzierte spezifische Stromstärke $i$ pro $\mathrm{rad} / \mathrm{h}$ in $\mathrm{n}$ Hexan $\left(20^{\circ} \mathrm{C}\right)$ in Abhängigkeit von der Dosisleistung $D$; Vergleich der gemessenen Werte mit dem theoretisch zu erwartenden Verlauf (Parameter: Feldstärke E) 
$d=1 \mathrm{~cm}$ und eine Feldstärke von $1 \mathrm{kV} / \mathrm{cm}$ eine untere Nachweisgrenze von $10 \mathrm{mrad} / \mathrm{h}$. Durch geeignete Nachreinigung des Hexans kann diese Grenze noch weiter reduziert werden, wobei man jedoch dann sehr empfindliche Gleichstrom-Meßverstärker verwenden muß.

Hinsichtlich Empfindlichkeit und Genauigkeit ist daher diese Methode vergleichbar mit der Glas- und Thermolumineszenzdosimetrie. Gegenüber den Methoden der chemischen Dosimetrie zeichnet sie sich durch eine wesentlich höhere Empfindlichkeit aus. Im Gegensatz zu diesen Methoden ermöglicht die Messung der strahleninduzierten Leitfähigkeit in Flüssigkeiten - ebenso wie die Dosimetrie mit gasgefüllten Ionisationskammern oder Proportionalzählrohren - eine direkte Messung der Dosisleistung.

Ein weiterer, vor allem für den Strahlenschutz wichtiger Vorteil dieser Methode besteht darin, daß sich die meisten dielektrischen Flüssigkeiten in Bezug auf die Absorption von sowohl $\gamma$ - als auch n-Strahlen in weiten Energiebereichen annähernd gewebeäquivalent verhalten. Abb. 6 zeigt dies am Beispiel des n-Hexans. Daraus geht hervor, da $\beta$ das Dosisverhältnis von Hexan zu weichem Gewebe bei $\gamma$-Strahlen von $0,1-10 \mathrm{MeV}$ Energie $1,05 \pm 0,05$ und bei $n$ Strahlen von $0,001-10 \mathrm{MeV}$ Energie $1,73 \pm 0,07$ ist; in diesen Energiebereichen verhält sich n-Hexan somit bis auf eine Abweichung von $\pm 5 \%$ gewebeäquivalent.

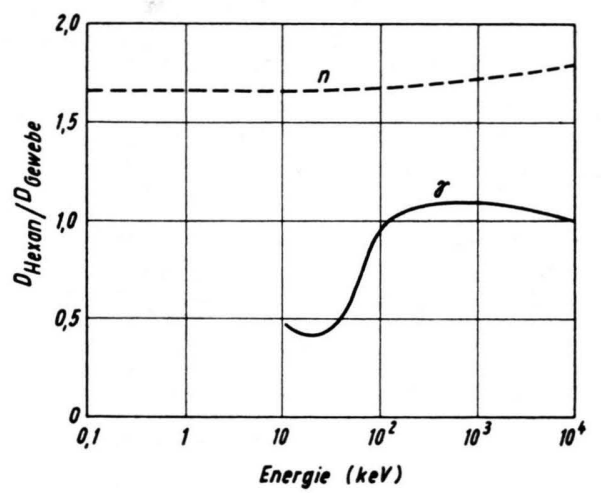

Abb. 6. Dosisverhältnis Hexan zu weichem Gewebe bei gleichem Fluens in Ahhängigkeit von der Energie für $\gamma$ - bzw. n-Strahlung.

\section{Abhängigkeit der Primärrekombination von der Art und Energie der Strahlung}

Die vorliegende Untersuchung zeigt am Beispiel des n-Hexans, daß der Einfluß der Primärrekombi- nation in dielektrischen Flüssigkeiten bei Einwirkung von $\mathrm{Co}^{60}-\gamma$-Strahlen mit Hilfe des Onsagerschen Ansatzes für die Rekombination eines einzelnen Ionenpaares in komprimierten Gasen erklärt werden kann. Die Voraussetzungen dieser Theorie sind jedoch auch für alle Strahlenarten erfüllt, bei denen die Cоuцомв-Wechselwirkung zwischen benachbarten Ionenpaaren in der Bahn des direkt ionisierenden Teilchens vernachlässigbar ist. Das heißt, die Entkommwahrscheinlichkeit $\varphi_{\mathrm{p}}$ in Bezug auf die Primärrekombination sollte unabhängig von der Art und Energie des Teilchens sein, solange der mittlere Abstand benachbarter Ionenpaare in der Teilchenbahn größer ist als der durch Gl. (1) definierte kritische Abstand $R_{\mathrm{k}} \quad\left(R_{\mathrm{k}}=300 \AA\right.$ in Hexan bei $\left.20{ }^{\circ} \mathrm{C}\right)$. Der mittlere Abstand benachbarter Ionenpaare kann mit Hilfe der Betheschen Theorie ermittelt werden. Geht man von der von LEA ${ }^{29}$ für Wasser der Dichte $1 \mathrm{~g} / \mathrm{cm}^{3}$ berechneten Zahl der Primärionisationen pro $\mu$ Bahnlänge für $\alpha$-Teilchen, Protonen und Elektronen verschiedener Energie aus, so folgt, daß in Hexan obige Bedingung für Elektronen-, RöNTGEN- und $\gamma$-Strahlen mit einer Energie größer als etwa $10 \mathrm{keV}$ und für Protonen mit einer Energie größer als etwa $10 \mathrm{MeV}$ erfültt ist.

Wertet man die von Ullmaier ${ }^{10}$ mit Mo-K $a$-Strahlung (Energie ca. $20 \mathrm{keV}$ ) durchgeführten Leitfähigkeitsmessungen in Hexan mit Hilfe der in der vorliegenden Arbeit beschriebenen Methode aus, so ergibt sich $\varphi_{\mathrm{po}} / W=0,0012 \mathrm{eV}^{-1}$ und $\bar{g}=0,07 \mathrm{~cm} / \mathrm{kV}$, d. h. innerhalb der Fehlergrenzen die gleichen Werte wie für $\mathrm{Co}^{60}-\gamma$-Strahlung (vgl. 3.2). Auch aus den Untersuchungen von $\mathrm{J}_{\text {ANuszaitis }}{ }^{9}$ an Hexan mit RöNTGEN- und $\gamma$-Strahlen im Energiebereich von $20 \mathrm{keV}-1,2 \mathrm{MeV}$ folgt, daß die Entkommwahrscheinlichkeit bei der Primärrekombination unabhängig von der Energie ist. Dieses Ergebnis stimmt mit den Folgerungen aus der Onsagerschen Theorie überein, während es im Widerspruch zur JAFFEschen Theorie der Kolonnenionisation steht.

Für $\alpha$-Teilchen, für Protonen mit Energien unterhalb $10 \mathrm{MeV}$ und dementsprechend auch für Neutronen ist hingegen eine LET-Abhängigkeit zu erwarten, da bei diesen Teilchen die Couloms-Wechselwirkung zwischen benachbarten Ionenpaaren in der Bahn der Teilchen nicht mehr vernachlässigbar ist. Da dann der mittlere, effektive Abstand $R_{\text {eff }}$ entgegengesetzt geladener Ionen mit zunehmender LET

29 D. E. Lea, Actions of Radiations on Living Cells, 2. Aufl., Cambridge Univ. Press, London 1956, 23-26. 
abnimmt, ist mit zunehmender LET eine Abnahme der Entkommwahrscheinlichkeit $\varphi_{\text {po }}$ und eine $\mathrm{Zu}$ nahme des Feldstärkefaktors $\bar{g}$ zu erwarten. Eine Auswertung der von Blanc et al. ${ }^{30}$ durchgeführten Leitfähigkeitsmessungen an Hexan bei Einwirkung von $\alpha$-Strahlen, Neutronen und $\gamma$-Strahlen bestätigt diese Folgerung.

Untersuchungen der strahleninduzierten Leitfähigkeit in dielektrischen Flüssigkeiten vermögen daher bei $\gamma$-Bestrahlung Aufschlüsse über die mittlere
Reichweite der beim Ionisationsprozeß entstehenden Elektronen und bei p-, n- und $\alpha$-Bestrahlung Aufschluß über die mittlere LET der Strahlung in Flüssigkeiten zu geben. Die Klärung dieser Fragen ist nicht nur für die Strahlenphysik, sondern auch für die Strahlenchemie und Strahlenbiologie von besonderem Interesse.

Herrn Prof. Dr. K. Lindenberger danken wir für wertvolle Diskussionen und sein Interesse an der Durchführung dieser Untersuchung.

30 D. Blanc, J. Mathieu u. P. Vermande, Health Phys. 11, 63 [1965].

\title{
Kernmagnetische Messungen von Selbstdiffusions-Koeffizienten in Wasser und Benzol bis zum kritischen Punkt
}

\author{
R. Hausser, G. Maier und F. Noack \\ I. Physikalisches Institut der Technischen Hochschule Stuttgart \\ (Z. Naturforschg. 21 a, 14]0--1415 [1966]; eingegangen am 12. Mai 1966)
}

\begin{abstract}
The most commonly used nuclear magnetic resonance spin-echo methods for determining selfdifiusion coefficients $D$ are described; some experimental data are presented for protons in water and in benzene from room temperature up to the critical point. The results are interpreted in terms of the free-volume rate theory of MAcEDo and Litovitz. The $D$-value of benzene may be described by a single activation energy. Water, however, shows such behavior only at high temperatures, thus demonstrating the strong influence of hydrogen bonding. The Sтокеs-Einstein relation between diffusion coefficient and viscosity is well satisfied over that part of the temperature range where viscosity values are available.
\end{abstract}

Mit Kernresonanz-Verfahren können Selbstdiffusions-Koeffizienten von Flüssigkeiten einfacher und schneller als mit Tracer-Methoden gemessen werden ${ }^{1}$. Ähnlich wie die kernmagnetischen Relaxationszeiten $T_{1}$ und $T_{2}$ liefert auch der Selbstdiffusions-Koeffizient $D$ Aufschlüsse über mikroskopische Substanzparameter und deren zeitliche und örtliche Korrelationsfunktionen ${ }^{2}$. $D$ kann beispielsweise mit der molekularen Reibungskonstante $\zeta$ verknüpft werden, die in den Theorien über Transportprozesse in Flüssigkeiten eine zentrale Rolle spielt ${ }^{3}$. Vielfach wird $D$ auch mit dem freien Volumen oder, ähnlich wie in Festkörpern, mit thermisch aktivierten Bewegungen über Potentialberge in Verbindung gebracht. Macedo und Litovitz ${ }^{4}$ haben diese beiden Aspekte in anschaulicher Weise vereinigt: ein Molekül kann nur dann einen Diffusionsschritt ausführen,

\footnotetext{
1 N. J. Trappeniers, C. J. Gerritsma u. P. H. Oosting, Phys. Letters 18, 256 [1965].

2 G. V. Chester, Rep. Prog. Phys. 26, 411 [1963].
}

wenn die thermische Energie zur Überwindung von Anziehungskräften der Nachbarn aufgebracht wird und gleichzeitig eine Leerstelle zur Verfügung steht. In diesem Fall gilt die Beziehung

$$
D=D_{0} \cdot e^{-\beta V_{0} / V_{\mathrm{F}}} \cdot e^{-E / R T}
$$

$\left(V_{0}\right.$ spezifisches Volumen bei dichtester Kugelpackung; $V$ spezifisches Volumen; $V_{\mathrm{F}}=V-V_{0}$ freies Volumen; $\beta$ Geometriefaktor zwischen 0,5 und $1 ; E$ Aktivierungsenergie; $R$ Gaskonstante; $T$ absolute Temperatur).

Die Kernresonanz-Messung von $D$ wird in einem Magnetfeld $\boldsymbol{H}=\boldsymbol{H}_{0}+\Delta \boldsymbol{H}$ ausgeführt, dessen Richtung über den Bereich des Probenvolumens näherungsweise konstant ist, während der Betrag linear vom Ort $r$ abhängen soll. Zur Realisierung wird dem für die Kernresonanz benötigten homogenen Magnetfeld $\boldsymbol{H}_{0}$ (meist einige $\mathrm{kOe}$ ) ein schwaches in-

\footnotetext{
3 B. H. Friedmann u. W. A. Steele, J. Chem. Phys. 40, 3669 [1964]. - P. Gray u. S. Rice, J. Chem. Phys. 40, 3671 [1964].

4 P. B. M:cedo u. T. A. Litovitz, J. Chem. Phys. 42, 245 [1965].
} 